PROCEEDINGS OF THE

AMERICAN MATHEMATICAL SOCIETY

Volume 126, Number 8, August 1998, Pages 2399-2406

S 0002-9939(98)04395-0

\title{
CONVERGENCE OF THE POINCARÉ SERIES FOR SOME CLASSICAL SCHOTTKY GROUPS
}

\author{
VLADIMIR MITYUSHEV
}

(Communicated by Albert Baernstein II)

\begin{abstract}
The Poincaré $\theta_{2}$-series for a multiply connected circular region can be either convergent or divergent absolutely. In this paper we prove a
\end{abstract} uniform convergence result for such a region.

\section{INTRODUCTION}

Let us consider mutually disjoint disks $D_{k}:=\left\{z \in \mathbb{C}:\left|z-a_{k}\right|<r_{k}\right\}(k=$ $1,2, \ldots, n)$ on the complex plane $\mathbb{C}$. We assume that $\overline{D_{k}} \cap \overline{D_{j}}=\emptyset$ for all $k \neq j$, where $\overline{D_{k}}$ is the closure of $D_{k}$. Let

$$
[k, z]:=r_{k}^{2} \backslash \overline{\left(z-a_{k}\right)}+a_{k}
$$

be an inversion with respect to the circumference $\partial D_{k}:=\left\{t \in \mathbb{C}:\left|t-a_{k}\right|=r_{k}\right\}$. Let us generate the Möbius transformations:

$$
\begin{gathered}
{\left[k_{1}, \ldots, k_{m} ; z\right]:=\left[k_{1}, \ldots, k_{m-1} ;\left[k_{m} ; z\right]\right], \text { where } k_{m}=1,2, \ldots, n ; k_{m-1}=1,2, \ldots, n ;} \\
k_{m} \neq k_{m-1} ; \ldots ; k_{2}=1,2, \ldots, n, k_{2} \neq k_{1} ; k_{1}=1,2, \ldots, n .
\end{gathered}
$$

Let $[k, U]$ denote a set which is symmetric to $U \subset \widehat{\mathbb{C}}:=\mathbb{C} \cup\{\infty\}$ with respect to $\left|t-a_{k}\right|=r_{k},\left[k_{1}, \ldots, k_{m} ; U\right]:=\left[k_{1}, \ldots, k_{m-1} ;\left[k_{m}, U\right]\right]$. We shall call $m$ the level of the transformation (1). The functions (1) with even level can be rewritten in the form $\gamma_{j}(z)=\left(\alpha_{j} z+b_{j}\right) /\left(c_{j} z+d_{j}\right), j=0,1, \ldots$, with $\alpha_{j} d_{j}-c_{j} b_{j}=1$. Here $\gamma_{0}(z):=$ $z, \gamma_{1}(z):=[1,2 ; z], \gamma_{2}(z):=[1,3 ; z], \ldots, \gamma_{n-1}(z):=[1, n ; z], \gamma_{n}(z):=[2,1 ; z]$ and so on. The numeration of the transformations is fixed in order of increasing level. The functions $\gamma_{j}(z)$ generate the Schottky group $\Gamma$ [1]-[3].

Let $H(z)$ be a meromorphic function in the extended complex plane $\widehat{\mathbb{C}}$. The Poincaré $\theta_{2 q}$-series [1], [2]

$$
\theta_{2 q}(z):=\sum_{j=0}^{\infty} H\left(\gamma_{j}(z)\right)\left(c_{j} z+d_{j}\right)^{-2 q} \quad(q \in \mathbb{Z} / 2)
$$

Received by the editors June 2, 1993 and, in revised form, November 17, 1995 and January 23, 1997.

1991 Mathematics Subject Classification. Primary 30E25, 30F40, 39B32.

(C) 1998 American Mathematical Society 
is associated with the group $\Gamma$. Here $z \in B:=\widehat{\mathbb{C}}-\left(B_{1} \cup \Lambda(\Gamma)\right), B_{1}$ is the set of poles of all $H\left(\gamma_{j}(z)\right)$ and $\gamma_{j}(z)$, and $\Lambda(\Gamma)$ is the limit set of $\Gamma$. When $q>1$ the series (2) converges absolutely and uniformly in every compact subset of $B$ [1], [2]. When $q=1$ the series (2) can be either absolutely convergent or absolutely divergent. It depends on the properties of $\Gamma$. Necessary and sufficient conditions for absolute and uniform convergence of the series have been found in [4], [5] in terms of the Hausdorff dimension of the limit set of $\Gamma$. Let us note that absolute and uniform convergencies was not studied separately in the previous papers.

Definition. A point $z$ is called a regular point of $\Gamma$ if there exist numbers $k_{1}, k_{2}, \ldots$, $k_{m}$ such that $\left[k_{1}, k_{2}, \ldots, k_{m} ; z\right]$ belongs to $\bar{D}$, where $D:=\widehat{\mathbb{C}}-\bigcup_{k=1}^{n} \overline{D_{k}}$ and the closure $\bar{D}$ is taken in $\widehat{\mathbb{C}}$.

In this paper we prove

Theorem 1. Let a rational function $H(z)$ have poles only at regular points of $\Gamma$. Then the Poincaré $\theta_{2}$-series converges uniformly in every compact subset of each region $\left[k_{1}, k_{2}, \ldots, k_{m} ; D\right] \cap B$. The order of summation depends on the region $\left[k_{1}, k_{2}, \ldots, k_{m} ; D\right]$.

Let us consider the Banach space $C$ consisting of the functions $\Phi(t):=\Phi_{k}(t)$, where $\left|t-a_{k}\right|=r_{k}(k=1,2, \ldots, n)$, which are continuous on $\bigcup_{k=1}^{n} \partial D_{k}$ with the norm $\|\Phi\|:=\max _{1 \leq k \leq n} \max _{\partial D_{k}}\left|\Phi_{k}(t)\right|$. And let us consider the closed subspace $C^{+} \in C$, for which the functions $\Phi_{k}(t)$ have analytic continuations to $D_{k}$.

The proof of Theorem 1 is based on the following

Main Lemma. The system of functional equations

$$
\Phi_{k}(z)=-\sum_{m=1}^{n}(\overline{[m, z]})^{\prime} \overline{\Phi_{m}([m, z])}+g_{k}(z),\left|z-a_{k}\right| \leq r_{k}, k=1,2, \ldots, n
$$

has a unique solution in $C^{+}$, where $g(z):=g_{k}(z),\left|z-a_{k}\right| \leq r_{k}(k=1,2, \ldots, n)$, is in $C^{+}$. That solution can be found by the method of successive approximations. The approximations converge in $C^{+}$.

Let the function $g_{k}(z)$ is meromorphic in $\left|z-a_{k}\right| \leq r_{k}(k=1,2, \ldots, n)$, i.e. $g_{k}(z)=s_{k}(z)+p_{k}(z)$, where $s_{k}(z)$ is analytic in $\left|z-a_{k}\right|<r_{k}$ and $p_{k}(z)$ is the principle part of $g_{k}(z)$. Let us consider the Banach space $C^{+}(p):=\left\{\Phi: \Phi-p_{k} \in\right.$ $\left.C^{+}, k=1,2, \ldots, n\right\}$ with the norm $\|\Phi\|_{C^{+}(p)}:=\left\|\Phi-p_{k}\right\|_{C^{+}}$. Following [11], we can consider (3) with $g \in C^{+}(p)$ for $\Phi \in C^{+}(q)$, where the rational functions $q_{k}(z)$ are constructed by $p_{k}(z)$. We show how $q_{k}(z)$ is constructed by $p_{k}(z)$ in Sec. 3

\section{Functional EQUations}

Before proving the main results let us prove some auxiliary facts about functional equations. For brevity we shall write (3) in the form $\Phi=A \Phi+g$ in $C^{+}$.

Let us define the shift operator $S_{m} \Phi_{m}(z)=\Phi_{m}([m, z])$ on $\left|t-a_{k}\right|=r_{k}$ as the integral operator

$$
S_{m} \Phi_{m}(z)=\frac{1}{2 \pi i} \int_{\partial D_{m}} \Phi_{m}(\tau) \frac{d \tau}{\tau-[m, t]},\left|t-a_{k}\right|=r_{k}, m=1,2, \ldots, n ; m \neq k .
$$


Let us consider the operator

$$
A_{C} \Phi(z)=-\sum_{m=1}^{n}(\overline{[m, t]})^{\prime} S_{m} \Phi_{m}(t),\left|t-a_{k}\right|=r_{k}, k=1,2, \ldots, n .
$$

It is easily seen that if $\Phi$ satisfies $\Phi=A \Phi+g$ in $C^{+}$, then $\Phi$ satisfies $\Phi=A_{C} \Phi+g$ in $C$. Conversely, if $g \in C^{+}$, then from the properties of $A_{C}$ for $\Phi \in C$ we obtain that $\Phi$ is a solution of $\Phi=A \Phi+g$ in $C^{+}$. Thus the equations $\Phi=A \Phi+g$ in $C$ and $\Phi=A_{C} \Phi+g$ in $C$ are equivalent for each $g \in C^{+}$.

Lemma 1. The homogeneous equation $\Phi=A \Phi$ in $C^{+}$has only the trivial solution.

Proof. If $\Phi_{m}(z)$ is a solution of the system

$$
\Phi_{k}(z)=-\sum_{m=1}^{n}(\overline{[m, z]})^{\prime} \overline{\Phi_{m}([m, z])},\left|z-a_{k}\right| \leq r_{k},
$$

then the $\Phi_{k}(z)$ are analytic in $\left|z-a_{k}\right| \leq r_{k}$. Let $\phi_{k}^{\prime}(z)=\Phi_{k}(z)$. Then integrating (4), we have

$$
\phi_{k}(z)=-\sum_{m=1}^{n} \overline{\phi_{m}([m, z])}+\gamma_{k},\left|z-a_{k}\right| \leq r_{k},
$$

where $\gamma_{k}$ are arbitrary constants of integration. Let us introduce the function

$$
\psi(z)=-\sum_{m=1}^{n} \overline{\phi_{m}([m, z])}
$$

analytic in $\bar{D}$. From (5) we obtain

$$
\psi(t)=\phi_{k}(t)-\overline{\phi_{k}(t)}-\gamma_{k},\left|t-a_{k}\right|=r_{k} .
$$

We shall rewrite the last relations in the form

$$
\begin{aligned}
\operatorname{Re} \psi(t) & =-\operatorname{Re} \gamma_{k}, \\
2 \operatorname{Im} \phi_{k}(t) & =\operatorname{Im} \psi(t)+\operatorname{Im} \gamma_{k},\left|t-a_{k}\right|=r_{k} .
\end{aligned}
$$

According to [9] the problem

$$
\operatorname{Re} \psi(t)=f(t)+c_{k},\left|t-a_{k}\right|=r_{k}, k=1,2, \ldots, n,
$$

is called the modified Dirichlet problem. Here, the required analytic function $\psi(z)$ is single-valued in $D, f(t)$ is a given Hölder continuous function, and the $c_{k}$ are undetermined constants. If one of the constants $c_{k}$ is fixed, for example $c_{1}$, then the problem (8) has a unique solution up to a purely imaginary additive constant $i \alpha$ [9]. If $f(t) \equiv 0$, then $\psi(z)=c_{1}+i \alpha, c_{k}=c_{1}(k=2,3, \ldots, n)$. This means that the problem (6) has only constant solutions. Hence, the right side of (7) is constant and the problem (7) with respect to the function $\phi_{k}(z)$ analytic in $\left|z-a_{k}\right|<r_{k}$ and continuous in $\left|z-a_{k}\right| \leq r_{k}$ has only constant solutions. Therefore, $\Phi_{k}(z)=$ $\phi_{k}^{\prime}(z) \equiv 0$.

The lemma is proved.

Lemma 2. The equation $\Phi=A_{C} \Phi+g$ is a Fredholm equation in $C$ and has a unique solution. 
Proof. The shift operator $S_{m} \Phi_{m}$ is compact in $C\left(\partial D_{m}\right)$. The operator of complex conjugation is bounded in $C$. Therefore the operator $A_{C}$ is compact in $C$, and the equation $\Phi=A_{C} \Phi+g$ is a Fredholm equation in $C$. If $g \in C^{+}$, then $\Phi \in C^{+}$. In particular, the zero function belongs to $C^{+}$. Hence by Lemma 1 the homogeneous equation in $C$ has only the trivial solution. Then, according to the Fredholm theorem, the nonhomogenious equation has a unique solution.

The lemma is proved.

Proof of the main lemma. By the properties of Fredholm equations [6] the spectrum of $A$ consists of eigenvalues only, and the condition $\rho(A)<1$ expresses the convergence of the method of successive approximations. Here $\rho(A)$ is the spectral radius of $A$. To prove the lemma it is sufficient to demonstrate that the equation $\Phi=\lambda A \Phi$ when $|\lambda| \leq 1$ has only the trivial solution. Let us note that the case $\lambda=1$ has been investigated in Lemma 1. Integrating the relations

$$
\Phi_{k}(z)=-\lambda \sum_{m=1}^{n}(\overline{[m, z]})^{\prime} \overline{\Phi_{m}([m, z])},\left|z-a_{k}\right| \leq r_{k}
$$

we obtain

$$
\phi_{k}(z)=-\lambda \sum_{m=1}^{n} \overline{\phi_{m}([m, z])}+c_{k},\left|z-a_{k}\right| \leq r_{k} .
$$

Here $\phi_{k}^{\prime}(z)=\Phi_{k}(z)$, and the $c_{k}$ are arbitrary constants. Let us introduce the function

$$
\psi(z)=-\lambda \sum_{m=1}^{n} \overline{\phi_{m}([m, z])}
$$

analytic in $\bar{D}$. From the above equalities we obtain the relations

$$
\psi(t)=\phi_{k}(t)-\lambda \overline{\phi_{k}(t)}-c_{k}, k=1,2, \ldots, n .
$$

According to [10] the problem (9) is called the boundary $\mathbb{R}$-linear problem. Here, the unknown functions $\psi(z)$ and $\phi_{k}(z)$ are analytic in $D$ and $D_{k}$, respectively, and continuous in $\bar{D}$ and $\overline{D_{k}}$. The function $\psi(z)$ is single-valued in $D$.

If $\lambda=\exp (2 i \Theta)$, i.e. $|\lambda|=1$, then the $\mathbb{R}$-linear problem (9) reduces to the modified Dirichlet problems

$$
\begin{aligned}
\operatorname{Re} \exp (-i \Theta) \psi(t) & =-\operatorname{Re} \exp (-i \Theta) c_{k},\left|t-a_{k}\right|=r_{k}, k=1,2, \ldots, n, \\
2 \operatorname{Im} \exp (-i \Theta) \phi_{k}(t) & =\operatorname{Im} \exp (-i \Theta)\left(\psi(t)+c_{k}\right),\left|t-a_{k}\right|=r_{k} .
\end{aligned}
$$

Like the problems (6) and (7), the last problems have only constant solutions.

If $|\lambda|<1$, then according to [7], [8] the homogeneous problem (9) (all $c_{k}=0$ ) has only one linearly independent solution. In this case we can write this solution as $\psi^{0}(z)=\beta, \phi_{k}^{0}(z)=(\beta+\lambda \bar{\beta}) /\left(1-|\lambda|^{2}\right)$, where $\beta$ is an arbitrary constant. According to [7], [8] the nonhomogeneous problem (9) (all $c_{k}$ are fixed) has the general solution $\psi(z)=\psi^{0}(z)+\psi^{1}(z), \phi_{k}(z)=\phi_{k}^{0}(z)+\phi_{k}^{1}(z)$, where $\psi^{0}(z), \phi_{k}^{0}(z)$ is the general solution of the homogeneous problem; $\psi^{1}(z), \phi_{k}^{1}(z)$ is a particular solution of the nonhomogenious problem. In this case we can write this solution as $\psi^{1}(z)=0 ; \phi_{k}^{1}(z)=\left(c_{k}+\lambda \overline{\beta c_{k}}\right) /\left(1-|\lambda|^{2}\right)$. 
Therefore, for $|\lambda| \leq 1$ the problem has only constant solutions. Hence, $\Phi_{k}(z)=$ $\phi_{k}^{\prime}(z) \equiv 0$.

The lemma is proved.

Applying the main lemma to $g_{k} / i$ instead of $g_{k}$, and setting $\Omega=i \Phi$, one obtains Lemma 3.

Lemma 3. The system of functional equations

$$
\Omega_{k}(z)=\sum_{m=1}^{n}(\overline{[m, z]})^{\prime} \overline{\Omega_{m}([m, z])}+g_{k}(z),\left|z-a_{k}\right| \leq r_{k}, k=1,2, \ldots, n,
$$

has a unique solution in $C^{+}$, where $g(z):=g_{k}(z),\left|z-a_{k}\right| \leq r_{k}(k=1,2, \ldots, n)$, is in $C^{+}$. That solution can be found by the method of successive approximations. The approximations converge in $C^{+}$.

\section{The Poincaré $\theta_{2}$-SERIES IN D}

Let us consider the systems (3) and (10) when $g_{k}(z)=-H(z)$. At first we assume that $H(z)$ has poles only in the region $D$. Let $\Phi_{k}(z)$ be a solution of (3) obtained by the method of successive approximation:

$$
\begin{aligned}
\Phi_{k}(z)= & -H(z)+\sum_{k_{1}=1 k_{1} \neq k}^{n}\left(\overline{\left[k_{1}, z\right]}\right)^{\prime} \overline{H\left(\left[k_{1}, z\right]\right)} \\
& -\sum_{k_{1}=1}^{n} \sum_{k_{1} \neq k}^{n}\left(\left[k_{2}=1 k_{2} \neq k_{1}, z\right]\right)^{\prime} H\left(\left[k_{2}, k_{1}, z\right]\right)+\ldots, \\
\left|z-a_{k}\right| \leq & r_{k} .
\end{aligned}
$$

The solution of (10) has the form

$$
\begin{aligned}
\Omega_{k}(z)= & -H(z)-\sum_{k_{1}=1 k_{1} \neq k}^{n}\left(\overline{\left[k_{1}, z\right]}\right)^{\prime} \overline{H\left(\left[k_{1}, z\right]\right)} \\
& -\sum_{k_{1}=1}^{n} \sum_{k_{1} \neq k}^{n}\left(\left[k_{2}, k_{1}, z\right]\right)^{\prime} H\left(\left[k_{2}, k_{1}, z\right]\right)-\ldots, \\
\left|z-a_{k}\right| \leq & r_{k} .
\end{aligned}
$$

These series converge in $C^{+}$, i.e. uniformly in $\left|z-a_{k}\right| \leq r_{k}$. Let us construct the functions

$$
\phi(z):=\sum_{k=1}^{n}(\overline{[k, z]})^{\prime} \overline{\Phi_{k}([k, z])}, \omega(z):=\sum_{k=1}^{n}(\overline{[k, z]})^{\prime} \overline{\Omega_{k}([k, z])} .
$$

Let us consider the generating elements of $\Gamma[m, k, z](k, m=1,2, \ldots n ; k \neq m)$. The equalities $\gamma_{j}^{\prime}(z)=\left(c_{j} z+d_{j}\right)^{-2}, \gamma_{j} \in \Gamma$, hold here [1], [2]. Hence, we have from (11)

$$
\frac{1}{2}[\phi(z)-\omega(z)]=\sum_{j=1}^{\infty} H\left[\gamma_{j}(z)\right]\left(c_{j} z+d_{j}\right)^{-2} .
$$


Remember that the order of summation is fixed in accordance with the level of $\gamma_{j} \in \Gamma$. Thus the Poincaré $\theta_{2}$-series (2) converges uniformly in $\bar{D}$, since

$$
\theta_{2}(z)=-\frac{1}{2}[(\phi(z)+H(z))-(\omega(z)-H(z))]
$$

Let us study system (3) for the functions $g_{k}(z)=-H(z)$ meromorphic in $\overline{D_{k}}$. Let $H(z)$ have a pole at a regular point $w$ belonging to some disk $\overline{D_{p}}$. Let us describe the process of removing this pole. Let $H(z)=H_{0}(z)+h(z)$, where $H_{0}(z)$ is analytic near $D_{p}$ and $h(z)$ is the principal part of $H(z)$ at $w$. Let us make the change of the $p$-th function $\Phi_{p}(z)=\Phi_{p}^{0}(z)-h(z)$. If $[p, w]$ doesn't belong to $\overline{D_{k}}$ for each $k \neq p$, then the process stops. If $[p, w]$ belongs to $\overline{D_{q}}$, then we make the change of the $q$-th function $\Phi_{q}(z)=\Phi_{q}^{0}(z)+(\overline{[p, z]})^{\prime} \overline{h([p, z])}$. If $[p, q, w]$ belongs to $\overline{D_{m}}$, then we make the next change, and so on. After a finite number of steps the process stops because $w$ is a regular point of $\Gamma$. Along similar lines we can make changes in accord with other poles of $H(z)$.

So we can construct a meromorphic function $q(z)$ such that the equation $\Phi=$ $A \Phi-H$ for $H \in C^{+}(p)$ with respect to $\Phi \in C^{+}(p)$ is reduced to the equation $\Psi=A \Psi-G$ in $C^{+}$. Here $p(z)$ is the principle part of $-H(z)$ in $\bigcup_{k=1}^{n} \overline{D_{k}}$, and the elements $\Psi=\Phi-q, G=H+q-A q$ belong to $C^{+}$. By virtue of the main lemma the series $\Psi=-\sum_{k=0}^{\infty} A^{k} G$ converges in $C^{+}$. This means that the series $\Phi=q+\Psi=q-\sum_{k=0}^{\infty} A^{k}(H+q-A q)$ converges uniformly in every compact subset of $\bigcup_{k=1}^{n} \overline{D_{k}} \backslash\left\{\right.$ poles of $q$ \}. Let us prove that the last series is $-\sum_{k=0}^{\infty} A^{k} H$. The finite sum $q-\sum_{k=0}^{N} A^{k}(H+q-A q)=-\sum_{k=0}^{N} A^{k} H+A^{N+1} q$. Since $q$ has poles only at regular points, there exists a number $M$ such that $A^{N+1} q \in C^{+}$. Therefore $A^{N+1} q \rightarrow 0$ as $N \rightarrow \infty$. The same argument is valid for the system (10) when the function $g_{k}(z)=-H(z)$ is meromorphic in $\overline{D_{k}}$. Using relations (11) and (12), let us construct the function

$$
\theta_{2}(z)=\sum_{j=1}^{\infty} H\left[\gamma_{j}(z)\right]\left(c_{j} z+d_{j}\right)^{-2},
$$

meromorphic in $\bar{D}$. This series converges uniformly in every compact subset of $\bar{D} \cap B$.

\section{The Poincaré Series as an aUtomorphic function}

Theorem 2. The Poincaré $\theta_{2}$-series (2) is an automorphic function:

$$
\theta_{2}(z)=\theta_{2}\left(\gamma_{j}(z)\right)\left(c_{j} z+d_{j}\right)^{-2} \text { for each } \gamma_{j} \text { from } \Gamma .
$$

The proof of the theorem in the case of absolute convergence is based on the change of the order of summation in (2) [1], [2]. In our case it is forbidden to change the order. Thus we present another proof of the theorem.

It follows from (11) that

$$
\phi(t)=-\overline{t-a_{k}} \overline{\Phi_{k}(t)}+\sum_{m=1}^{n}(\overline{[m, t]})^{\prime} \overline{\Phi_{m}([m, t])},\left|t-a_{k}\right|=r_{k},
$$


since $(\overline{[k, t]})^{\prime}=-\frac{\overline{t-a_{k}}}{t-a_{k}},\left|t-a_{k}\right|=r_{k}$. Using (3), we calculate

$$
\begin{aligned}
& \operatorname{Im}\left(t-a_{k}\right) \phi(t) \\
& \quad=\operatorname{Re}\left(t-a_{k}\right)\left[\Phi_{k}(t)+\sum_{m=1 m \neq k}^{n}(\overline{[m, t]})^{\prime} \overline{\Phi_{m}([m, t])}\right] \\
& \quad=\operatorname{Im}\left(t-a_{k}\right) H(t),\left|t-a_{k}\right|=r_{k} .
\end{aligned}
$$

The region $[k ; D]$ is symmetric to the region $D$ with respect to $\left|t-a_{k}\right|=r_{k}$. The circumference $\left[k ; \partial D_{m}\right]$ is symmetric to the circumference $\left|t-a_{m}\right|=r_{m}$ with respect to $\left|t-a_{k}\right|=r_{k}(m \neq k)$, and the region $[k, m ; D]$ is symmetric to the region $[k ; D]$ with respect to $\left[k ; \partial D_{m}\right]$. Let us note that the numbers $k$ and $m$ are fixed in the definitions of these regions; $D \cup \partial D_{k} \cup[k ; D]$ and $[k ; D] \cup\left[k ; \partial D_{m}\right] \cup[k, m ; D]$ are the fundamental regions of $\Gamma$. The relation (14) implies meromorphic continuation of $(\phi(z)+H(z))$ to $[k ; D]$ and $[k, m ; D]$. Using the reflection principle we have

$$
\begin{aligned}
\phi(z)+H(z) & =-(\overline{[k, z]})^{\prime}[\overline{\phi([k, z])}+\overline{H([k, z])}], z \in[k, D], \\
\phi(z)+H(z) & =\gamma_{p}^{\prime}(z)\left[\phi\left(\gamma_{p}(z)\right)+H\left(\gamma_{p}(z)\right)\right], z \in[k, m, D],
\end{aligned}
$$

where $\gamma_{p}(z)$ is the composition of symmetries with respect to $\left[k ; \partial D_{m}\right]$ and $\partial D_{k}$. The transformation $\gamma_{p}(z)$ is an element of the group $\Gamma$.

Along similar lines the relation

$$
\operatorname{Re}\left(t-a_{k}\right)[\omega(t)-H(t)]=0, \quad\left|t-a_{k}\right|=r_{k}, k=1,2, \ldots, n,
$$

holds. Hence, the function $[\omega(z)-H(z)]$ can be meromorphically continued to $[k ; D]$ and $[k, m ; D]$, by

$$
\begin{aligned}
\omega(z)-H(z) & =(\overline{[k, t]})^{\prime}[\overline{\omega([k, t])}-\overline{H([k, t])}], z \in[k, D], \\
\omega(z)-H(z) & =\gamma_{p}^{\prime}(z)\left[\omega\left(\gamma_{p}(z)\right)-H\left(\gamma_{p}(z)\right)\right], z \in[k, m, D] .
\end{aligned}
$$

It follows from $(12)$ that the function $\theta_{2}(z)$ can be meromorphically continued to $[k ; D]$ and $[k, m ; D]$. Moreover, the values of $\theta_{2}(z)$ in $D$ and $[k, m ; D]$ are related by the equality

$$
\theta_{2}(z)=\theta_{2}\left(\gamma_{p}(z)\right) \gamma_{p}^{\prime}(z), z \in[k, m ; D] \cap B=\gamma_{p}(D \cap B) .
$$

Using the reflection principle, we can continue $\theta_{2}(z)$ through $\left[k ; \partial D_{m}\right]$ to the next symmetric domain, and so on to the region of discontinuity of $\Gamma$. Moreover the values of $\theta_{2}(z)$ in $\widehat{\mathbb{C}}-\{$ poles $\}$ are related by the equality (13).

Theorem 2 is proved.

It follows from the proof of Theorem 2 that $\phi(z)$ and $\omega(z)$ are represented as series in each $\left[k_{1}, \ldots, k_{m} ; D\right]$. Substituting these series into $(12)$, we obtain a $\theta_{2}$-series with another order of summation. Thus $\theta_{2}(z)$ can be represented as the series

$$
\sum_{j=1}^{\infty} H\left[\gamma_{\sigma(j)}(z)\right] \gamma_{\sigma(j)}^{\prime}(z)
$$

in each image $\left[k_{1}, \ldots, k_{m} ; D\right]$ of $D$ under the mapping $z \rightarrow\left[k_{1}, \ldots, k_{m} ; z\right]$, where $\sigma$ is the bijection of the set of all non-negative integers that corresponds canonically to $\left[k_{1}, \ldots, k_{m} ; D\right]$.

This proves Theorem 1 . 
The function $\theta_{2}(z)$ can be applied to construct the Schwarz operator (Green's function) for a circular multiply connected region in analytic form. This was done in the papers [12], [13].

\section{ACKNOWLEDGMENT}

The author would like to thank the referee for many valuable comments.

\section{REFERENCES}

[1] H.Poincaré, Oeuvres, Gauthier-Villar, Paris t.2, 1916; t.4, 1950; t.9, 1954. MR 97c:01053

[2] L.Ford, Automorphic functions, New York, 1929.

[3] A.F.Berdon, Geometry of discrete groups, Springer-Verlag, New York, 1986. MR 87h:20014

[4] T.Akaza, Singular sets of some Kleinian groups, Nagoya Math J 26 (1966), 127-143. MR 34:7794

[5] T.Akaza, K.Inoue, Limit sets of geometrically finite free Kleinian groups, Tohoku Math J 36 (1984), 1-16. MR 85b:30058

[6] M.A.Krasnoselski, et al., Approximate solution of operator equations, Nauka, Moscow, 1969; English transl., Wolters-Noordhoff, Groningen, 1974. MR 41:4271; MR 52:6515

[7] B.Bojarski, On a boundary value problem of function theory, Soobschenia AN Gruz. SSR 25 (1960), 385-390. (Russian)

[8] L.G.Mikhajlov, A new class of singular integral equations, Wolters-Noordhoff Publ., Groningen 1970. MR 41:8812

[9] F.D.Gakhov, Boundary value problems, Perg.Press, Oxford, 1966. MR 33:6311

[10] V.Mityushev, A method of functional equations for boundary value problems of continuous media, Reports Math Phys 33 (1993), 137-147. MR 95e:30038

[11] V.Mityushev, Solution of linear functional equation with shift into domain in class of analytic functions, Izvestia AN BSSR. Ser. Phys.-Math. 5 (1983) 117. (Russian)

[12] V.Mityushev, Solution of the Hilbert boundary value problem for a multiply connected domain, Slupskie Prace Mat.-Przyr. 9a (1994), 37-69. MR 96d:30050

[13] V.Mityushev, Hilbert boundary value problem for multiply connected domains, Complex Variables [to appear]

Department of Mathematics, Pedagogical College, ul.Arciszewskiego 22B, 76-200 SLuPSK, POLAND 